\title{
Stabilisation of Hybrid Stochastic Differential Equations by Feedback Control based on Discrete-time Observations of State and Mode
}

\author{
Gongfei Song ${ }^{1}$, Bo-Chao Zheng ${ }^{2}$, Qi Luo ${ }^{3}$, Xuerong $\mathrm{Mao}^{4, *}$ \\ ${ }^{1}$ CICAEET, School of Information and Control, Nanjing University of Information Science and \\ Technology, Nanjing, Jiangsu, 210044, China \\ ${ }^{2}$ CICAEET, School of Information and Control, Nanjing University of Information Science and \\ Technology, Nanjing, Jiangsu, 210044, China \\ ${ }^{3}$ CICAEET, School of Information and Control, Nanjing University of Information Science and \\ Technology, Nanjing, Jiangsu, 210044, China \\ ${ }^{4}$ Department of Mathematics and Statistics, University of Strathclyde, Glasgow G1 1XH, UK \\ *corresponding: x.mao@strath.ac.uk
}

\begin{abstract}
Mao [10] recently initiated the study of the mean-square exponential stabilisation of continuous-time hybrid stochastic differential equations (SDEs) by the feedback controls based on the discrete-time observations of the state. However, the feedback controls still depend on the continuous-time observations of the mode. Of course this is perfectly fine if the mode of the system is obvious (i.e. fully observable at no cost). However, it could often be the case where the mode is not obvious and it costs to identify the current mode of the system. To reduce the control cost, it is reasonable we identify the mode at the discrete times when we make observations for the state. Hence the feedback control should be designed based on the discrete-time observations of both state and mode. The aim of this paper is to show how to design such a feedback control to stabilise a given hybrid SDE.
\end{abstract}

Keywords: stabilisation, feedback control, discrete-time observations, hybrid stochastic differential equations, Brownian motion.

\section{Introduction}

Mao [10] recently initiated the study of the mean-square exponential stabilisation of continuoustime hybrid stochastic differential equations (SDEs) (also known as SDEs with Markovian switching [14]) by feedback controls based on the discrete-time observations of the state. To make it clear why we need to further study this stabilisation problem, let us make a quick review on the problem studied in [10].

Throughout this paper, unless otherwise specified, we will use the same notation as in [10]. Consider a hybrid SDE

$$
d x(t)=f(x(t), r(t), t) d t+g(x(t), r(t), t) d w(t)
$$

on $t \geq t_{0}(\geq 0)$, where $f: R^{n} \times S \times R_{+} \rightarrow R^{n}, g: R^{n} \times S \times R_{+} \rightarrow R^{n \times m}, w(t)=$ $\left(w_{1}(t), \cdots, w_{m}(t)\right)^{T}$ is an $m$-dimensional Brownian motion and $r(t)$ is a Markov chain with its state space $S=\{1,2, \cdots, N\}$ and the generator $\Gamma=\left(\gamma_{i j}\right)_{N \times N}$. Moreover, $x(t) \in R^{n}$ is known 
as the state while $r(t)$ as the mode of the system (see e.g. [8, 14]). The hybrid SDEs have been used to model real-world systems where they may experience abrupt changes in their structure and parameters in addition to uncertainties. For example, consider the well-known Black-Scholes SDE model in finance $d x(t)=\mu x(t) d t+\sigma x(t) d w(t)$ (known as the geometric Brownian motion too, see e.g. [7]), where Black and Scholes assumed that the rate of return $\mu$ and the volatility $\sigma$ are constants. However, it has been proved by many authors that both of them are random processes in many situations. There is a strong evidence to indicate that the rate $\mu$ is a Markov jump process which can be modelled by a Markov chain as it depends very much on the interest rate. Of course, when the rate jumps, the volatility will jump accordingly. Taking these jumps into account, the Black-Scholes SDE model has recently be generalised to form a new financial model $d x(t)=\mu(r(t)) x(t) d t+\sigma(r(t)) x(t) d w(t)$. Another typical example is the hybrid SDE model in power systems (see e.g. [18]). For further references on hybrid SDEs, we mention, for example, $[5,12,15,17,20])$. In particular, [8] is one of most cited papers (more than 550 Google citations) while [14] is the first book in this area (more than 800 Google citations).

Suppose that the given hybrid SDE (1) is unstable. Instead of using a classical continuoustime feedback control $u(x(t), r(t), t)$ which requires continuous observations of the state $x(t)$ for all time $t \geq 0$ (see e.g. [5, 11, 13, 19, 20]), Mao proposed in [10] to design a feedback control $u\left(x\left(\delta\left(t, t_{0}, \tau\right)\right), r(t), t\right)$ based on the discrete-time observations of the state in order to make the controlled system

$$
d x(t)=\left(f(x(t), r(t), t)+u\left(x\left(\delta\left(t, t_{0}, \tau\right)\right), r(t), t\right)\right) d t+g(x(t), r(t), t) d w(t)
$$

become exponentially stable in mean square. Here $\tau>0$ and

$$
\delta\left(t, t_{0}, \tau\right)=t_{0}+\left[\left(t-t_{0}\right) / \tau\right] \tau
$$

in which $\left[\left(t-t_{0}\right) / \tau\right]$ is the integer part of $\left(t-t_{0}\right) / \tau$. The advantage of such a discrete-time feedback control requires only state observations $x\left(t_{0}+k \tau\right)$ at discrete times $t_{0}, t_{0}+\tau, t_{0}+2 \tau, \cdots$ and hence it will cost much less than a continuous-time feedback control. As pointed out in [10], although the corresponding problem for the deterministic differential equations has been studied by several authors (see e.g. [1, 3, 4]), Mao [10] is the first paper that addresses the stabilisation problem by discrete-time feedback controls for hybrid SDEs.

We are now in the position to explain why we need this paper to continue the study initiated by [10]. Observe that the discrete-time feedback control in the controlled SDE (2) is based on the discrete-time observations of the state, $x\left(t_{0}+k \tau\right)(k=0,1,2 \cdots)$ but it still depends on the continuous-time observations of the mode, $r(t)$ on $t \geq t_{0}$. Of course this is perfectly fine if the mode of the system is obvious (i.e. fully observable at no cost), for example, in a financial system where the mode represents the interest rate (see e.g. [21, 22]). However, it could often be the case where the mode is not obvious and it costs to identify the current mode of the system (see e.g. $[6,15])$. To reduce the control cost, it is reasonable that we identify the mode at the same discrete times $t_{0}+k \tau(k \geq 0)$ when we make observations for the state. It is in this spirit that we write this paper to consider an $n$-dimensional controlled hybrid SDE

$$
\begin{aligned}
d z(t) & =\left[f(z(t), r(t), t)+u\left(z\left(\delta\left(t, t_{0}, \tau\right)\right), r\left(\delta\left(t, t_{0}, \tau\right)\right), t\right)\right] d t \\
& +g(z(t), r(t), t) d w(t)
\end{aligned}
$$

on $t \geq t_{0}$, where our new feedback control is based on the discrete-time observations of both state $z\left(t_{0}+k \tau\right)$ and mode $r\left(t_{0}+k \tau\right)$. We will compare the discrete-time controlled SDE (4) 
with the continuous-time controlled SDE (10). We will show that if the SDE (10) is mean-square exponentially stable, then so is the SDE (4) provided $\tau$ is sufficiently small. We will form our main result in Section 2. We will then present several lemmas in Section 3 in order to prove Theorem 1 in Section 4. We will also present some case studies in Section 5 and conclude our paper in Section 6.

Before we develop our new theory, we should emphasise that the mathematical analysis in this paper is much more complicated than that in [10] due to the difficulties arisen from the discretetime observations $r\left(t_{0}+k \tau\right)$ of the mode. We will point out these difficulties in detail later when we perform the proofs of our theory. In other words, this paper is not a simple generalisation of Mao [10] but needs new techniques.

\section{Statement of Main Result}

To study the stability of the discrete-time controlled system (4), we impose a standing hypothesis for this paper.

Assumption 1. Assume that the coefficients of the system and the control function

$$
\begin{aligned}
f, u & : R^{n} \times S \times R_{+} \rightarrow R^{n}, \\
g & : R^{n} \times S \times R_{+} \rightarrow R^{n \times m}
\end{aligned}
$$

are Borel measurable. Assume also that there are positive constants $K_{1}, K_{2}, K_{3}$ such that

$$
\begin{aligned}
& |f(x, i, t)-f(y, i, t)| \leq K_{1}|x-y| \\
& |u(x, i, t)-u(y, i, t)| \leq K_{2}|x-y| \\
& |g(x, i, t)-g(x, i, t)| \leq K_{3}|x-y|
\end{aligned}
$$

for all $(x, y, i, t) \in R^{n} \times R^{n} \times S \times R_{+}$. Moreover,

$$
f(0, i, t)=0, \quad u(0, i, t)=0, \quad g(0, i, t)=0
$$

for all $(i, t) \in S \times R_{+}$.

It should be pointed out that condition (6) is for the stability purpose of this paper and condition (5) is for the existence and uniqueness of the solution (see e.g.[7, 14, 16]). We also see that these conditions imply the following linear growth condition

$$
\begin{gathered}
|f(x, i, t)| \leq K_{1}|x|, \quad|u(x, i, t)| \leq K_{2}|x|, \\
|g(x, i, t)| \leq K_{3}|x|
\end{gathered}
$$

for all $(x, i, t) \in R^{n} \times S \times R_{+}$.

We claim that under Assumption 1, for any initial data $z\left(t_{0}\right)=z_{0} \in L_{\mathcal{F}_{t_{0}}}^{2}\left(R^{n}\right)$ and $r\left(t_{0}\right)=$ $r_{0} \in M_{\mathcal{F}_{t_{0}}}(S)$ at time $t_{0} \geq 0$, equation (4) has a unique solution $z(t)$ such that $\mathbb{E}|z(t)|^{2}<\infty$ for all $t \geq t_{0}$. In fact, it is known (see e.g. [14, page 112]) that $\left\{r\left(\delta\left(t, t_{0}, \tau\right)\right)\right\}_{t \geq t_{0}}=\left\{r\left(t_{0}+k \tau\right)\right\}_{k \geq 0}$ is a discrete-time Markov chain with the one-step transition probability matrix $e^{\tau \Gamma}$. Moreover, for $t \in\left[t_{0}, t_{0}+\tau\right]$, equation (4) becomes a hybrid SDE

$$
\begin{aligned}
d z(t) & =\left[f(z(t), r(t), t)+u\left(z\left(t_{0}\right), r\left(t_{0}\right), t\right)\right] d t \\
& +g(z(t), r(t), t) d w(t) .
\end{aligned}
$$


It is hence known (see e.g. [14, Theorem 3.8 on page 81]) that this SDE has a unique solution $z(t)$ on $\left[t_{0}, t_{0}+\tau\right]$ such that $\mathbb{E}|z(t)|^{2}<\infty$. Repeating this procedure on time intervals $\left[t_{0}+k \tau,(k+1) \tau\right]$ for $k \geq 1$, we see what we have claimed.

To emphasise the role of the initial data, we will denote the solution by $z\left(t ; z_{0}, r_{0}, t_{0}\right)$ and the Markov chain by $r\left(t ; r_{0}, t_{0}\right)$. The arguments in the last paragraph shows that the process has the following property at the discrete times $t_{0}+k \tau(k \geq 0)$

$$
\begin{gathered}
\left(z\left(t ; z_{0}, r_{0}, t_{0}\right), r\left(t ; r_{0}, t_{0}\right)\right)=\left(z\left(t ; z\left(t_{0}+k \tau\right), r\left(t_{0}+k \tau\right), t_{0}+k \tau\right),\right. \\
\left.r\left(t ; r\left(t_{0}+k \tau\right), t_{0}+k \tau\right)\right),
\end{gathered}
$$

for all $t \geq t_{0}+k \tau$, where $z\left(t_{0}+k \tau\right)=z\left(t_{0}+k \tau ; z_{0}, r_{0}, t_{0}\right)$ and $r\left(t_{0}+k \tau\right)=r\left(t_{0}+k \tau ; r_{0}, t_{0}\right)$.

We will relate our discrete-time controlled system (4) with the corresponding continuous-time controlled system

$$
\begin{aligned}
d y(t) & =(f(y(t), r(t), t)+u(y(t), r(t), t)) d t \\
& +g(y(t), r(t), t) d w(t)
\end{aligned}
$$

on $t \geq t_{0}$ with initial data $y\left(t_{0}\right)=z_{0} \in L_{\mathcal{F}_{t_{0}}}^{2}\left(R^{n}\right)$ and $r\left(t_{0}\right)=r_{0} \in M_{\mathcal{F}_{t_{0}}}(S)$ at time $t_{0} \geq 0$. It is known (see e.g. [14, Theorem 3.8 on page 81]) that under Assumption 1, equation (10) has a unique solution, denoted by $y\left(t ; z_{0}, r_{0}, t_{0}\right)$ such that $\mathbb{E}\left|y\left(t ; z_{0}, r_{0}, t_{0}\right)\right|^{2}<\infty$ for all $t \geq t_{0}$. Our aim here is to show that if system (10) is mean-square exponentially stable, then so is the discrete-time controlled system (4) provided $\tau$ is sufficiently small. In other words, our new theory enables us to transfer the discrete-time controlled problem (4) into the classical continuous-time controlled problem (10).

In section 5, we will present some case studies to demonstrate how to design the control function $u: R^{n} \times S \times R_{+} \rightarrow R^{n}$ for system (10) to be mean-square exponentially stable. But in this section, we simply impose the following assumption.

Assumption 2. Assume that there is a pair of positive constants $M$ and $\gamma$ such that the solution of the continuous-time controlled SDE (10) satisfies

$$
\mathbb{E}\left|y\left(t ; z_{0}, r_{0}, t_{0}\right)\right|^{2} \leq M \mathbb{E}\left|z_{0}\right|^{2} e^{-\gamma\left(t-t_{0}\right)} \quad \forall t \geq t_{0}
$$

for all $t_{0} \geq 0, z_{0} \in L_{\mathcal{F}_{t_{0}}}^{2}\left(R^{n}\right)$ and $r_{0} \in M_{\mathcal{F}_{t_{0}}}(S)$.

The following theorem shows that this same control function also makes the discrete-time controlled system (4) to be mean-square exponentially stable as long as $\tau$ is sufficiently small (namely we make observations on both state and mode frequently enough).

Theorem 1. Let Assumptions 1 and 2 hold. Let $\varepsilon \in(0,1)$ be a free parameter. Let $\bar{\tau}>0$ be the unique root to the equation

$$
\bar{H}(\bar{\tau}, \varepsilon)=0.5(1-\varepsilon)
$$

where

$$
\begin{aligned}
\bar{H}(\tau, \varepsilon) & =\frac{\tau K_{2}\left[4 \tau\left(K_{1}^{2}+K_{2}^{2}\right)+2 K_{3}^{2}\right]+4 K_{2}\left(1-e^{-\hat{\gamma} \tau}\right)}{2 K_{1}+2 K_{2}+K_{3}^{2}} \\
& \times e^{\left(2 K_{1}+4 K_{2}+K_{3}^{2}\right)(\tau+\log (2 M / \varepsilon) / \gamma)} \\
& \times\left[e^{\left(2 K_{1}+2 K_{2}+K_{3}^{2}\right)(\tau+\log (2 M / \varepsilon) / \gamma)}-1\right]
\end{aligned}
$$


in which

$$
\hat{\gamma}=\max _{i \in S}\left(-\gamma_{i i}\right)
$$

If $\tau<\bar{\tau}$, then there is a pair of positive constants $\bar{M}$ and $\lambda$ such that the solution of the controlled hybrid SDE (4) satisfies

$$
\mathbb{E}\left|z\left(t ; z_{0}, r_{0}, t_{0}\right)\right|^{2} \leq \bar{M} \mathbb{E}\left|z_{0}\right|^{2} e^{-\lambda\left(t-t_{0}\right)} \forall t \geq t_{0}
$$

for all $t_{0} \geq 0, z_{0} \in L_{\mathcal{F}_{t_{0}}}^{2}\left(R^{n}\right)$ and $r_{0} \in M_{\mathcal{F}_{t_{0}}}(S)$. That is, the controlled hybrid SDE (4) is mean-square exponentially stable.

Please note that $\varepsilon$ is a free parameter in $(0,1)$. By choosing different parameter $\varepsilon$, it is possible to get better $\bar{\tau}$. This is different from [10]. Unfortunately, we still do not know how to choose $\varepsilon$ in order to maximise $\bar{\tau}$.

\section{Lemmas}

To prove Theorem 1, we present a number of lemmas in this section. We first recall that $r(t)$ is a right-continuous Markov chain with the state space $S=\{1,2, \cdots, N\}$ and the generator $\Gamma=\left(\gamma_{i j}\right)_{N \times N}$. In particular, we recall that $-\gamma_{i i}=\sum_{j \neq i} \gamma_{i j}>0$. It is known that almost all sample paths of $r(t)$ are step-functions (or piecewise-constant functions) with a finite number of simple jumps in any finite subinterval of $R_{+}$. The following lemma estimates the probability of jumps.

Lemma 1. For any $t \geq 0, v>0$ and $i \in S$,

$$
\mathbb{P}(r(s) \neq i \text { for some } s \in[t, t+v] \mid r(t)=i) \leq 1-e^{-\hat{\gamma} v},
$$

where $\hat{\gamma}$ has been defined by (14) in the statement of Theorem 1 .

Proof. Given $r(t)=i$, define the stopping time

$$
\rho_{i}=\inf \{s \geq t: r(s) \neq i\},
$$

where and throughout this paper we set $\inf \emptyset=\infty$ (in which $\emptyset$ denotes the empty set as usual). It is well known (see e.g. [2]) that $\rho_{i}-t$ has the exponential distribution with parameter $-\gamma_{i i}$. Hence

$$
\begin{aligned}
& \mathbb{P}(r(s) \neq i \text { for some } s \in[t, t+v] \mid r(t)=i) \\
= & \mathbb{P}\left(\rho_{i}-t \leq v \mid r(t)=i\right)=\int_{0}^{v} \frac{1}{-\gamma_{i i}} e^{\gamma_{i i} s} d s \\
= & 1-e^{\gamma_{i i} v} \leq 1-e^{-\hat{\gamma} v}
\end{aligned}
$$

as desired.

In the following lemmas, we will write $z\left(t ; z_{0}, r_{0}, t_{0}\right)=z(t), y\left(t ; z_{0}, r_{0}, t_{0}\right)=y(t)$ and $\delta\left(t, t_{0}, \tau\right)=$ $\delta_{t}$ for convenience.

Lemma 2. Let Assumption 1 hold. Then for any initial data $z_{0}, r_{0}, t_{0}$, the solution of equation (4) satisfies

$$
\mathbb{E}|z(t)|^{2} \leq \mathbb{E}\left|z_{0}\right|^{2} e^{\left(2 K_{1}+2 K_{2}+K_{3}^{2}\right)\left(t-t_{0}\right)}
$$


and

$$
\begin{aligned}
\mathbb{E}\left|z(t)-z\left(\delta_{t}\right)\right|^{2} \leq & \mathbb{E}\left|z_{0}\right|^{2} \tau\left[4 \tau\left(K_{1}^{2}+K_{2}^{2}\right)+2 K_{3}^{2}\right] \\
& \times e^{\left(2 K_{1}+2 K_{2}+K_{3}^{2}\right)\left(t-t_{0}\right)}
\end{aligned}
$$

for all $t \geq t_{0}$.

The proof of this lemma is standard (see e.g. [10]) so is omitted. The following lemma will play a central role in the proof of Theorem 1. The proof of this lemma is very technical and shows clearly the difficulty due to the discrete-time observations on the mode.

Lemma 3. Let Assumption 1 hold. Then for any initial data $z_{0}, r_{0}, t_{0}$,

$$
\begin{aligned}
& \mathbb{E}|z(t)-y(t)|^{2} \\
& \leq \mathbb{E}\left|z_{0}\right|^{2} H(\tau) e^{\left(2 K_{1}+4 K_{2}+K_{3}^{2}\right)\left(t-t_{0}\right)} \\
& \times\left[e^{\left(2 K_{1}+2 K_{2}+K_{3}^{2}\right)\left(t-t_{0}\right)}-1\right]
\end{aligned}
$$

for all $t \geq t_{0}$, where

$$
H(\tau)=\frac{\tau K_{2}\left[4 \tau\left(K_{1}^{2}+K_{2}^{2}\right)+2 K_{3}^{2}\right]+4 K_{2}\left(1-e^{-\hat{\gamma} \tau}\right)}{2 K_{1}+2 K_{2}+K_{3}^{2}} .
$$

Proof. The Itô formula shows that for $t \geq t_{0}$,

$$
\begin{aligned}
\mathbb{E}|z(t)-y(t)|^{2} \\
=\mathbb{E} \int_{t_{0}}^{t}\left[2(z(s)-y(s))^{T}\right. \\
\times(f(z(s), r(s), s)-f(y(s), r(s), s) \\
\left.+u\left(z\left(\delta_{s}\right), r\left(\delta_{s}\right), s\right)-u(y(s), r(s), s)\right) \\
\left.+|g(z(s), r(s), s)-g(y(s), r(s), s)|^{2}\right] d s .
\end{aligned}
$$

By Assumption 1, we then have

$$
\mathbb{E}|z(t)-y(t)|^{2} \leq \mathbb{E} \int_{t_{0}}^{t}\left(2 K_{1}+K_{3}^{2}\right)|z(s)-y(s)|^{2} d s+J(t),
$$

where

$$
\begin{aligned}
J(t)= & \mathbb{E} \int_{t_{0}}^{t} 2(z(s)-y(s))^{T} \\
& \times\left[u\left(z\left(\delta_{s}\right), r\left(\delta_{s}\right), s\right)-u(y(s), r(s), s)\right] d s \\
= & \mathbb{E} \int_{t_{0}}^{t} 2(z(s)-y(s))^{T} \\
\times & \left(\left[u\left(z\left(\delta_{s}\right), r\left(\delta_{s}\right), s\right)-u\left(z\left(\delta_{s}\right), r(s), s\right)\right]\right. \\
& +\left[u\left(z\left(\delta_{s}\right), r(s), s\right)-u(z(s), r(s), s)\right] \\
& +[u(z(s), r(s), s)-u(y(s), r(s), s)]) d s .
\end{aligned}
$$


Using Assumption 1, we get

$$
\begin{aligned}
J(t) & \leq K_{2} \mathbb{E} \int_{t_{0}}^{t}\left[4|z(s)-y(s)|^{2}+\left|z\left(\delta_{s}\right)-z(s)\right|^{2}\right] d s \\
& +K_{2}^{-1} J_{1}(t)
\end{aligned}
$$

where

$$
J_{1}(t)=\mathbb{E} \int_{t_{0}}^{t}\left|u\left(z\left(\delta_{s}\right), r\left(\delta_{s}\right), s\right)-u\left(z\left(\delta_{s}\right), r(s), s\right)\right|^{2} d s .
$$

Substituting (22) into (21) yields

$$
\begin{aligned}
& \mathbb{E}|z(t)-y(t)|^{2} \\
\leq & \left(2 K_{1}+4 K_{2}+K_{3}^{2}\right) \int_{t_{0}}^{t} \mathbb{E}|z(s)-y(s)|^{2} d s \\
+ & K_{2} \int_{t_{0}}^{t} \mathbb{E}\left|z\left(\delta_{s}\right)-z(s)\right|^{2} d s+K_{2}^{-1} J_{1}(t) .
\end{aligned}
$$

But, by Lemma 2, we have

$$
\begin{aligned}
\int_{t_{0}}^{t} \mathbb{E} \mid z\left(\delta_{s}\right) & -\left.z(s)\right|^{2} d s \leq \frac{\tau\left[4 \tau\left(K_{1}^{2}+K_{2}^{2}\right)+2 K_{3}^{2}\right]}{2 K_{1}+2 K_{2}+K_{3}^{2}} \\
& \times \mathbb{E}\left|z_{0}\right|^{2}\left[e^{\left(2 K_{1}+2 K_{2}+K_{3}^{2}\right)\left(t-t_{0}\right)}-1\right] .
\end{aligned}
$$

To estimate $J_{1}(t)$, let $h=h(t)$ be the integer part of $\left(t-t_{0}\right) / \tau$ and define $t_{k}=t_{0}+k \tau$ for $k=0,1,2, \cdots$. Then, by the well-known Fubini theorem, we have

$$
\begin{array}{rl}
J_{1}(t)=\sum_{k=0}^{h} \int_{t_{k}}^{t \wedge t_{k+1}} & \mathbb{E} \mid u\left(z\left(t_{k}\right), r\left(t_{k}\right), s\right) \\
& -\left.u\left(z\left(t_{k}\right), r(s), s\right)\right|^{2} d s .
\end{array}
$$

By Assumption 1, we can derive that, for $t_{k} \leq s \leq t \wedge t_{k+1}$,

$$
\begin{aligned}
& \mathbb{E}\left|u\left(z\left(t_{k}\right), r\left(t_{k}\right), s\right)-u\left(z\left(t_{k}\right), r(s), s\right)\right|^{2} \\
&= \mathbb{E}\left[\mathbb{E}\left(\left|u\left(z\left(t_{k}\right), r\left(t_{k}\right), s\right)-u\left(z\left(t_{k}\right), r(s), s\right)\right|^{2} \mid \mathcal{F}_{t_{k}}\right)\right] \\
& \leq \mathbb{E}\left[4 K_{2}^{2}\left|z\left(t_{k}\right)\right|^{2} \mathbb{E}\left(I_{\left\{r(s) \neq r\left(t_{k}\right)\right\}} \mid \mathcal{F}_{t_{k}}\right)\right] \\
&= \mathbb{E}\left[4 K_{2}^{2}\left|z\left(t_{k}\right)\right|^{2} \mathbb{E}\left(\sum_{i \in S} I_{\left\{r\left(t_{k}\right)=i\right\}} I_{\{r(s) \neq i\}} \mid \mathcal{F}_{t_{k}}\right)\right] \\
&= \mathbb{E}\left[4 K_{2}^{2}\left|z\left(t_{k}\right)\right|^{2} \sum_{i \in S} I_{\left\{r\left(t_{k}\right)=i\right\}}\right. \\
&\left.\times \mathbb{P}\left(r(s) \neq i \mid r\left(t_{k}\right)=i\right)\right] .
\end{aligned}
$$


But, by Lemma 1,

$$
\begin{aligned}
& \mathbb{P}\left(r(s) \neq i \mid r\left(t_{k}\right)=i\right) \\
\leq & \mathbb{P}\left(r(\bar{s}) \neq i \text { for some } \bar{s} \in\left[t_{k}, t \wedge t_{k+1} \mid r\left(t_{k}\right)=i\right)\right. \\
\leq & 1-e^{-\hat{\gamma} \tau} .
\end{aligned}
$$

Hence

$$
\begin{aligned}
& \mathbb{E}\left|u\left(z\left(t_{k}\right), r\left(t_{k}\right), s\right)-u\left(z\left(t_{k}\right), r(s), s\right)\right|^{2} \\
\leq & \mathbb{E}\left[4 K_{2}^{2}\left|z\left(t_{k}\right)\right|^{2}\left(1-e^{-\hat{\gamma} \tau}\right)\right] \\
= & 4 K_{2}^{2}\left(1-e^{-\hat{\gamma} \tau}\right) \mathbb{E}\left|z\left(t_{k}\right)\right|^{2} .
\end{aligned}
$$

Substituting this into (25), we get

$$
J_{1}(t) \leq 4 K_{2}^{2}\left(1-e^{-\hat{\gamma} \tau}\right) \sum_{k=0}^{h} \int_{t_{k}}^{t \wedge t_{k+1}} \mathbb{E}\left|z\left(t_{k}\right)\right|^{2} d s .
$$

But, by Lemma 2, we then have

$$
\begin{aligned}
& \sum_{k=0}^{h} \int_{t_{k}}^{t \wedge t_{k+1}} \mathbb{E}\left|z\left(t_{k}\right)\right|^{2} d s \\
\leq & \sum_{k=0}^{h} \int_{t_{k}}^{t \wedge t_{k+1}} \mathbb{E}\left|z_{0}\right|^{2} e^{\left(2 K_{1}+2 K_{2}+K_{3}^{2}\right)\left(t_{k}-t_{0}\right)} d s \\
\leq & \sum_{k=0}^{h} \int_{t_{k}}^{t \wedge t_{k+1}} \mathbb{E}\left|z_{0}\right|^{2} e^{\left(2 K_{1}+2 K_{2}+K_{3}^{2}\right)\left(s-t_{0}\right)} d s \\
= & \mathbb{E}\left|z_{0}\right|^{2} \int_{t_{0}}^{t} e^{\left(2 K_{1}+2 K_{2}+K_{3}^{2}\right)\left(s-t_{0}\right)} d s \\
= & \frac{\mathbb{E}\left|z_{0}\right|^{2}}{2 K_{1}+2 K_{2}+K_{3}^{2}}\left[e^{\left(2 K_{1}+2 K_{2}+K_{3}^{2}\right)\left(t-t_{0}\right)}-1\right] .
\end{aligned}
$$

Putting this into (28) gives

$$
\begin{aligned}
J_{1}(t) \leq & \frac{4 K_{2}^{2}\left(1-e^{-\hat{\gamma} \tau}\right)}{2 K_{1}+2 K_{2}+K_{3}^{2}} \mathbb{E}\left|z_{0}\right|^{2} \\
& \times\left[e^{\left(2 K_{1}+2 K_{2}+K_{3}^{2}\right)\left(t-t_{0}\right)}-1\right] .
\end{aligned}
$$

Substituting (24) and (29) into (23), we get

$$
\begin{aligned}
& \mathbb{E}|z(t)-y(t)|^{2} \\
\leq & \left(2 K_{1}+4 K_{2}+K_{3}^{2}\right) \int_{t_{0}}^{t} \mathbb{E}|z(s)-y(s)|^{2} d s \\
+ & H(\tau) \mathbb{E}\left|x_{0}\right|^{2}\left[e^{\left(2 K_{1}+2 K_{2}+K_{3}^{2}\right)\left(t-t_{0}\right)}-1\right] .
\end{aligned}
$$

Finally, the well-known Gronwall inequality implies the desired assertion (20). 


\section{Proof of Theorem 1}

Let us now begin to prove our main Theorem 1. We first observe that for any fixed $\varepsilon \in(0,1)$, $\bar{H}(\tau, \varepsilon)$ is an increasing function of $\tau \geq 0$ with $\bar{H}(0, \varepsilon)=0$ and $\bar{H}(\tau, \varepsilon) \rightarrow \infty$ as $\tau \rightarrow \infty$. So there must be a unique root $\bar{\tau}>0$ to equation (12). Fix initial data $z_{0}, r_{0}, t_{0}$ arbitrarily and write $z\left(t ; z_{0}, r_{0}, t_{0}\right)=z(t)$ and $r\left(t ; r_{0}, t_{0}\right)=r(t)$ simply. For $k=0,1,2, \cdots$, we write $t_{0}+k \tau=t_{k}$, $z\left(t_{0}+k \tau\right)=z_{k}$ and $z\left(t_{0}+k \tau\right)=r_{k}$. Recalling property (9), we see that

$$
z(t)=z\left(t ; z_{k}, r_{k}, t_{k}\right) \forall t \geq t_{k}
$$

In other words, when $t \geq t_{k}$, we may regard $z(t)$ as the solution of equation(4) with initial data $z\left(t_{k}\right)=z_{k}$ and $r\left(t_{k}\right)=r_{k}$ at time $t_{k}$.

Let us choose a positive integer $\bar{k}$ such that

$$
\frac{\log (2 M / \varepsilon)}{\gamma \tau} \leq \bar{k}<\frac{\log (2 M / \varepsilon)}{\gamma \tau}+1
$$

So

$$
2 M e^{-\gamma \bar{k} \tau} \leq \varepsilon
$$

For $i=0,1,2, \cdots$, let $y_{(i+1) \bar{k}}=y\left((i+1) \bar{k} ; z_{i \bar{k}}, r_{i \bar{k}}, t_{i \bar{k}}\right)$. Clearly, we have

$$
\mathbb{E}\left|z_{(i+1) \bar{k}}\right|^{2} \leq 2 \mathbb{E}\left|y_{(i+1) \bar{k}}\right|^{2}+2 \mathbb{E}\left|z_{(i+1) \bar{k}}-y_{(i+1) \bar{k}}\right|^{2} .
$$

But, by Assumption 2 and (33), we have

$$
\mathbb{E}\left|y_{(i+1) \bar{k}}\right|^{2} \leq M e^{-\gamma \bar{k} \tau} \mathbb{E}\left|x_{i \bar{k}}\right|^{2} \leq 0.5 \varepsilon \mathbb{E}\left|x_{i \bar{k}}\right|^{2} .
$$

By (31), we have $z_{(i+1) \bar{k}}=z\left((i+1) \bar{k} ; z_{i \bar{k}}, r_{i \bar{k}}, t_{i \bar{k}}\right)$. Hence, by Lemma 3 and inequality (32),

$$
\begin{aligned}
& \mathbb{E}\left|z_{(i+1) \bar{k}}-y_{(i+1) \bar{k}}\right|^{2} \\
\leq & \mathbb{E}\left|z_{i \bar{k}}\right|^{2} H(\tau) e^{\left(2 K_{1}+4 K_{2}+K_{3}^{2}\right) \bar{k} \tau} \\
\times & {\left[e^{\left(2 K_{1}+2 K_{2}+K_{3}^{2}\right) \bar{k} \tau}-1\right] } \\
\leq & \mathbb{E}\left|z_{i \bar{k}}\right|^{2} H(\tau) e^{\left(2 K_{1}+4 K_{2}+K_{3}^{2}\right)(\tau+\log (2 M / \varepsilon) / \gamma)} \\
\times & {\left[e^{\left(2 K_{1}+2 K_{2}+K_{3}^{2}\right)(\tau+\log (2 M / \varepsilon) / \gamma)}-1\right] . }
\end{aligned}
$$

Recalling the definition of $\bar{H}(\varepsilon, \tau)$ (i.e. (13) ) along with the definition of $H(\tau)$ in the statement of Lemma 3, we see that

$$
\mathbb{E}\left|z_{(i+1) \bar{k}}-y_{(i+1) \bar{k}}\right|^{2} \leq \mathbb{E}\left|z_{i \bar{k}}\right|^{2} \bar{H}(\tau, \varepsilon) .
$$

Substituting (35) and (37) into (34) yields

$$
\mathbb{E}\left|z_{(i+1) \bar{k}}\right|^{2} \leq(\varepsilon+2 \bar{H}(\tau, \varepsilon)) \mathbb{E}\left|z_{i \bar{k}}\right|^{2} .
$$

Since $\tau<\bar{\tau}$ and $\bar{H}(\tau, \varepsilon)$ is an increasing function of $\tau$, we see from (12) that

$$
\varepsilon+2 \bar{H}(\tau, \varepsilon)<1 .
$$


We may hence write

$$
\varepsilon+2 \bar{H}(\tau, \varepsilon)=e^{-\lambda \bar{k} \tau}
$$

for some $\lambda>0$. It then follows from (38) that

$$
\mathbb{E}\left|z_{(i+1) \bar{k}}\right|^{2} \leq \mathbb{E}\left|z_{i \bar{k}}\right|^{2} e^{-\lambda \bar{k} \tau} .
$$

This implies immediately that

$$
\mathbb{E}\left|z_{i \bar{k}}\right|^{2} \leq \mathbb{E}\left|z_{0}\right|^{2} e^{-\lambda i \bar{k} \tau}, \quad \forall i=0,1,2, \cdots .
$$

Now, for any $t \geq t_{0}$, there is a unique $i \geq 0$ such that $t_{0}+i \bar{k} \tau \leq t<t_{0}+(i+1) \bar{k} \tau$. By (31), we have

$$
z(t)=z\left(t ; z_{i \bar{k}}, r_{i \bar{k}}, t_{i \bar{k}}\right)
$$

Hence, by Lemma 2 and (40),

$$
\begin{aligned}
\mathbb{E}|z(t)|^{2} & \leq \mathbb{E}\left|z_{i \bar{k}}\right|^{2} e^{\left(2 K_{1}+2 K_{2}+K_{3}^{2}\right) \bar{k} \tau} \\
& \leq \mathbb{E}\left|z_{0}\right|^{2} e^{-\lambda i \bar{k} \tau+\left(2 K_{1}+2 K_{2}+K_{3}^{2}\right) \bar{k} \tau} \\
& \leq \bar{M} \mathbb{E}\left|z_{0}\right|^{2} e^{-\lambda\left(t-t_{0}\right)}
\end{aligned}
$$

where $\bar{M}=e^{\lambda \tau+\left(2 K_{1}+2 K_{2}+K_{3}^{2}\right) \bar{k} \tau}$. But this is the required assertion (15). We have therefore proved that the controlled system(4) is mean-square exponentially stable. The proof is complete.

\section{Case Studies}

The new theory established above enables us to transfer the stabilisation problem (4) by a discretetime feedback control to the stabilisation problem (10) by a continuous-time feedback control. To use our new theory, we need to know how to design the control function $u: R^{n} \times S \times R_{+} \rightarrow$ $R^{n}$ such that the hybrid SDE (10) is mean-square exponentially stable, namely Assumption 2 is satisfied. There is an intensive literature in the area of stability of hybrid SDEs, for example, $[5,11,13,19,20]$. For the convenience of the reader, we state a lemma which follows easily from [14, Thereom 4.5 on page 166].

Lemma 4. Assume that there are symmetric positive-definite matrices $Q_{i}, i \in S$, as well as a positive number $\gamma$, such that

$$
L Q(y, i, t) \leq-\gamma y^{T} Q_{i} y
$$

for all $(y, i, t) \in R^{n} \times S \times R_{+}$, where the function $L Q: R^{n} \times S \times R_{+} \rightarrow R$ is defined by

$$
\begin{aligned}
& L Q(y, i, t):=2 y^{T} Q_{i}[f(y, t, i)+u(y, t, i)] \\
& +\operatorname{trace}\left[g^{T}(y, i, t) Q_{i} g(y, i, t)\right]+\sum_{j=1}^{N} \gamma_{i j} y^{T} Q_{j} y .
\end{aligned}
$$

Then the solution of the SDE (10) satisfies

$$
\mathbb{E}\left|y\left(t ; z_{0}, r_{0}, t\right)\right|^{2} \leq M \mathbb{E}\left|z_{0}\right|^{2} e^{-\gamma\left(t-t_{0}\right)}, \quad \forall t \geq t_{0}
$$

for all $t_{0} \geq 0, z_{0} \in L_{\mathcal{F}_{t_{0}}}^{2}\left(R^{n}\right)$ and $r_{0} \in M_{\mathcal{F}_{t_{0}}}(S)$, where

$$
M=\frac{\max _{i \in S} \lambda_{\max }\left(Q_{i}\right)}{\min _{i \in S} \lambda_{\min }\left(Q_{i}\right)} .
$$

We now apply this lemma to establish a couple of useful results on the design of the control function $u: R^{n} \times S \times R_{+} \rightarrow R^{n}$. 


\subsection{Linear hybrid SDEs}

Suppose that we are given an $n$-dimensional linear hybrid SDE

$$
d x(t)=A(r(t)) x(t) d t+\sum_{k=1}^{m} B_{k}(r(t)) x(t) d w_{k}(t)
$$

on $t \geq t_{0}$. Here $A, B_{k}$ are mappings from $S \rightarrow R^{n \times n}$ and we will write $A(i)=A_{i}$ and $B_{k}(i)=$ $B_{k i}$. The linear hybrid SDEs of form (44) appear frequently in many branches of science and industry, for example, finance $[7,21,22]$ and engineering $[6,15,18]$. It is often that a given hybrid $\mathrm{SDE}$ is unstable and we are required to design a feedback control function, in the structure form of $u(x, i)=F(i) G(i) x$ in the drift part, so that the controlled SDE

$$
\begin{aligned}
d x(t) & =\left[A(r(t)) x(t)+F\left(r\left(\delta_{t}\right)\right) G\left(r\left(\delta_{t}\right)\right) x\left(\delta_{t}\right)\right] d t \\
& +\sum_{k=1}^{m} B_{k}(r(t)) x(t) d w_{k}(t),
\end{aligned}
$$

will be mean-square exponentially stable. Here $\delta_{t}=\delta\left(t, t_{0}, \tau\right), F$ and $G$ are mappings from $S$ to $R^{n \times l}$ and $R^{l \times n}$, respectively, and in general $l<n$. The advantage of the feedback control $F\left(r\left(\delta_{t}\right)\right) G\left(r\left(\delta_{t}\right)\right) x\left(\delta_{t}\right)$ based on the discrete-time observations of state $x\left(\delta_{t}\right)$ and mode $r\left(\delta_{t}\right)$ has been explained in Section 1, in comparison with the continuous-time feedback control $F(r(t)) G(r(t)) x(t)$. We here use the structure control of the form $F\left(r\left(\delta_{t}\right)\right) G\left(r\left(\delta_{t}\right)\right) x\left(\delta_{t}\right)$ instead of the general form $u\left(x\left(\delta_{t}\right), r\left(\delta_{t}\right), t\right)$ is because that such type of controls is one of the most popular feedback controls for linear systems (see e.g. $[1,4,5,11,15]$ ).

We will write $F(i)=F_{i}$ and $G(i)=G_{i}$. We only consider the case where $G_{i}$ 's are given but $F_{i}$ 's are to be designed. This is the situation where we can observe the output $y\left(\delta_{t}\right):=$ $G\left(r\left(\delta_{t}\right)\right) x\left(\delta_{t}\right)$ and the mode $r\left(\delta_{t}\right)$ at the discrete time $\delta_{t}$ while we know the output matrices $G_{i}$ but we cannot observe the state $x\left(\delta_{t}\right)$. Making use of the output and the mode, we need to design matrices $F_{i}$ to form the structure feedback control $F\left(r\left(\delta_{t}\right)\right) G\left(r\left(\delta_{t}\right)\right) x\left(\delta_{t}\right)$ in order to stabilise the system. The following corollary describes how $F_{i}$ 's can be designed.

Corollary 1. Assume that for some number $\gamma>0$, there is a set of solutions $Q_{i} \in R^{n \times n}$ and $Y_{i} \in R^{n \times l}(i \in S)$, with $Q_{i}=Q_{i}^{T}>0$, to following linear matrix inequalities (LMIs)

$$
\begin{aligned}
& Q_{i} A_{i}+Y_{i} G_{i}+A_{i}^{T} Q_{i}+G_{i}^{T} Y_{i}^{T} \\
& +\sum_{k=1}^{m} B_{k i}^{T} Q_{i} B_{k i}+\sum_{j=1}^{N} \gamma_{i j} Q_{j}+\gamma Q_{i} \leq 0 .
\end{aligned}
$$

Let $M$ be defined by (43) and set

$$
\begin{gathered}
K_{1}=\max _{i \in S}\left\|A_{i}\right\|, \quad K_{2}=\max _{i \in S}\left\|Q_{i}^{-1} Y_{i} G_{i}\right\|, \\
K_{3}=\max _{i \in S} \sqrt{\sum_{k=1}^{m}\left\|B_{k i}\right\|^{2}} .
\end{gathered}
$$

Choose a free parameter $\varepsilon \in(0,1)$ and let $\bar{\tau}>0$ be the unique root to equation (12). If we set $F_{i}=Q_{i}^{-1} Y_{i}(i \in S)$ and make sure that $\tau<\bar{\tau}$, then the controlled hybrid SDE (45) is mean-square exponentially stable. 
Proof. To apply Theorem 1, we consider the corresponding controlled hybrid SDE based on the continuous-time observations of both state and mode in the form

$$
\begin{aligned}
d y(t) & =[A(r(t))+F(r(t)) G(r(t))] y(t) d t \\
& +\sum_{k=1}^{m} B_{k}(r(t)) y(t) d w_{k}(t)
\end{aligned}
$$

on $t \geq t_{0}$ with the initial data $y\left(t_{0}\right)=z_{0} \in L_{\mathcal{F}_{t_{0}}}^{2}\left(R^{n}\right)$ and $r\left(t_{0}\right)=r_{0} \in M_{\mathcal{F}_{t_{0}}}(S)$. Noting that $F_{i}=Q_{i}^{-1} Y_{i}$, we can easily verify that

$$
\begin{aligned}
L Q(y, i, t) & =y^{T}\left[Q_{i} A_{i}+Y_{i} G_{i}+A_{i}^{T} Q_{i}+G_{i}^{T} Y_{i}^{T}\right. \\
& \left.+\sum_{k=1}^{m} B_{k i}^{T} Q_{i} B_{k i}+\sum_{j=1}^{N} \gamma_{i j} Q_{j}\right] y .
\end{aligned}
$$

Using condition (46), we then obtain that

$$
L Q(y, i, t) \leq-\gamma y^{T} Q_{i} y
$$

By Lemma 4, we see that the solution of equation (48) satisfies

$$
\mathbb{E}\left|y\left(t ; z_{0}, r_{0}, t\right)\right|^{2} \leq M \mathbb{E}\left|z_{0}\right|^{2} e^{-\gamma\left(t-t_{0}\right)}, \quad \forall t \geq t_{0} .
$$

That is, Assumption 2 is fulfilled. Moreover, Assumption 1 is clearly fulfilled with $K_{1}, K_{2}, K_{3}$ defined by (47). The corollary therefore follows from Theorem 1 immediately. $\square$.

\subsection{Nonlinear Hybrid SDEs}

Let us now consider another special case of the controlled hybrid SDE (4) where we will use a linear structure feedback control of the form $u(x, i)=F(i) G(i) x$. That is, the controlled hybrid SDE (4) reduces to

$$
\begin{aligned}
d x(t) & =\left[f(x(t), r(t), t)+F\left(r\left(\delta_{t}\right)\right) G\left(r\left(\delta_{t}\right)\right) x\left(\delta_{t}\right)\right] d t \\
& +g(x(t), r(t), t) d w(t) .
\end{aligned}
$$

Here $\delta_{t}=\delta\left(t, t_{0}, \tau\right), F$ and $G$ are the same as in Section 5.1. We once again only consider the case where $G_{i}$ 's are given but $F_{i}$ 's are to be designed. Of course, we need to keep the global Lipschitz condition on the coefficients $f$ and $g$ (corresponding to Assumption 1). More precisely, we impose the following assumption.

Assumption 3. Assume that there are positive constants $K_{1}, K_{3}$ such that

$$
\begin{aligned}
|f(x, i, t)-f(y, i, t)| & \leq K_{1}|x-y| \\
|g(x, i, t)-g(x, i, t)| & \leq K_{3}|x-y|
\end{aligned}
$$

for all $(x, y, i, t) \in R^{n} \times R^{n} \times S \times R_{+}$. Moreover,

$$
f(0, i, t)=0, \quad g(0, i, t)=0
$$

for all $(i, t) \in S \times R_{+}$. 
We will need another assumption.

Assumption 4. For each $i \in S$, there is a pair of symmetric $n \times n$-matrices $Q_{i}$ and $\bar{Q}_{i}$ with $Q_{i}=Q_{i}^{T}>0$ such that

$$
\begin{aligned}
2 x^{T} Q_{i} f(x, i, t) & +\operatorname{trace}\left[g^{T}(x, i, t) Q_{i} g(x, i, t)\right] \\
& \leq x^{T} \bar{Q}_{i} x
\end{aligned}
$$

for all $(x, i, t) \in R^{n} \times S \times R_{+}$.

The following corollary describes how the state feedback control $u(x, i)=F_{i} G_{i} x$ can be designed by designing $F_{i}$ 's when $G_{i}$ 's are given.

Corollary 2. Let Assumptions 4 and 3 hold. Assume that for some number $\gamma>0$, there is a set of solutions $Y_{i} \in R^{n \times l}(i \in S)$ to the following LMIs

$$
\bar{Q}_{i}+Y_{i} G_{i}+G_{i}^{T} Y_{i}^{T}+\sum_{j=1}^{N} \gamma_{i j} Q_{j}+\gamma Q_{i} \leq 0
$$

Define $M$ by (43) and set

$$
K_{2}=\max _{i \in S}\left\|Q_{i}^{-1} Y_{i} G_{i}\right\|
$$

Let $\bar{\tau}>0$ be the unique root to equation (12). If we set $F_{i}=Q_{i}^{-1} Y_{i}(i \in S)$ and make sure that $\tau<\bar{\tau}$, then the controlled hybrid SDE (50) is mean-square exponentially stable.

Proof. Once again, to apply Theorem 1, we consider the corresponding controlled hybrid SDE based on the continuous-time observations of both state and mode in the form

$$
\begin{aligned}
d y(t) & =[f(y(t), r(t), t)+F(r(t)) G(r(t)) y(t)] d t \\
& +g(y(t), r(t), t) d w(t)
\end{aligned}
$$

on $t \geq t_{0}$ with initial data $y\left(t_{0}\right)=z_{0} \in L_{\mathcal{F}_{t_{0}}}^{2}\left(R^{n}\right)$ and $r\left(t_{0}\right)=r_{0} \in M_{\mathcal{F}_{t_{0}}}(S)$. Using $F_{i}=Q_{i}^{-1} Y_{i}$, Assumption 4 and condition (54), we can derive that

$$
\begin{aligned}
& L Q(y, i, t)=2 y^{T} Q_{i}\left[f(y, i, t)+F_{i} G_{i} y\right] \\
& +\operatorname{trace}\left[g^{T}(y, i, t) Q_{i}(g(y, i, t)]+\sum_{j=1}^{N} \gamma_{i j} y^{T} Q_{j} y\right. \\
& \leq y^{T}\left[\bar{Q}_{i}+Y_{i} G_{i}+G_{i}^{T} Y_{i}^{T}+\sum_{j=1}^{N} \gamma_{i j} Q_{j}\right] y \\
& \leq-\gamma y^{T} Q_{i} y .
\end{aligned}
$$

By Lemma 4, we see that the solution of equation (56) satisfies

$$
\mathbb{E}\left|y\left(t ; z_{0}, r_{0}, t\right)\right|^{2} \leq M \mathbb{E}\left|z_{0}\right|^{2} e^{-\gamma\left(t-t_{0}\right)}, \quad \forall t \geq t_{0}
$$

That is, Assumption 2 is fulfilled. By Assumption 3 as well as the form of the feedback control, we also see that Assumption 1 is fulfilled with $K_{1}$ and $K_{3}$ being specified in Assumption 3 and $K_{2}$ being defined by (55). Consequently, the corollary follows from Theorem 1 directly. 


\section{Conclusion and Further Comments}

In this paper we have shown clearly that unstable hybrid SDEs can be stabilised by the feedback controls based on the discrete-time observations of state and mode. We have also presented two case studies to demonstrate how the state feedback control functions can be designed to stabilise the given unstable hybrid SDEs.

The key condition imposed in this paper is the global Lipschtiz condition. Although this condition covers some important hybrid SDEs including the linear ones as we discussed in the previous section, it is somehow restrictive as there are other nonlinear hybrid SDEs in real world whose coefficients are only locally Lipschitz continuous e.g. the population dynamical systems [7]. On the other hand, the technique and method used in this paper could not easily be modified to cope with the local Lipschitz case. New techniques and methods will be needed. We should also point out that to make our theory more understandable as well as to avoid complicated notations, we have only considered the controlled hybrid SDE (4), where the discrete-time control is designed in the drift part. It is useful and interesting to consider the case where the discrete-time control is designed in the diffusion part, or different discrete-time controls are designed in the drift and diffusion parts. Furthermore, we have assumed in this paper that all the components of the state $x(t)$ are observable at the discrete times. However, it is often the case where only partial components of the state are observable. It is therefore interesting and useful to design the feedback control based on the partially observed state. However, due to the page limit here, we will report these results elsewhere.

\section{Acknowledgments}

The authors would like to thank the referees and editors for their very professional comments and suggestions. The authors would also like to thank the Leverhulme Trust (RF-2015-385), the Natural Science Foundation of China $(61503190,61403207,61273155)$, the Natural Science Funds of Jiangsu Province (BK20131000, BK20150927), the outstanding innovation team project of universities in Jiangsu province, and the Ministry of Education (MOE) of China (MS2014DHDX020) for their financial support. In particular, X. Mao would like to thank the Royal Society of London for his Royal Society Wolfson Research Merit Award (WM160014).

\section{References}

[1] Allwright, J.C., Astolfi, A., Wong, H.P.: 'A note on asymptotic stabilisation of linear systems by periodic, piecewise constant, output feedback', Automatica, 2015, 41, (2), pp. 339-344

[2] Anderson, W.J.: 'Continuous-time Markov chains' (Springer, New York, 1991)

[3] Ebihara, Y., Yamaguchi, J., Hagiwara, T.: 'Periodically time-varying controller synthesis for multiobjective $H_{2} / H_{\infty}$ control of discrete-time systems and analysis of achievable performance', Syst. Control Lett., 2011, 60, (9), pp. 709-717

[4] Chen, T., Francis, B.: 'Optimal sampled data control systems' (Springer-Verlag, London, 1995) 
[5] Ji, Y., Chizeck, H.J.: 'Controllability, stabilisability and continuous-time Markovian jump linear quadratic control', IEEE Trans. Autom. Control, 1990, 35, (7), pp. 777-788

[6] Krishnamurthy, V., Wang, X., Yin, G.: 'Spreading code optimisation and adaptation in CDMA via discrete stochastic approximation', IEEE Trans. Inf. Theory, 2004, 50, (9), pp. 1927-1949

[7] Mao, X.: 'Stochastic differential equations and their applications' (2nd Edition, Chichester: Horwood Pub., 2007)

[8] Mao, X.: 'Stability of stochastic differential equations with Markovian switching', Sto. Proc. Their Appl., 1999, 79, (1), pp. 45-67

[9] Mao, X.: 'Exponential stability of stochastic delay interval systems with Markovian switching', IEEE Trans. Autom. Control, 2002, 47, (10), pp. 1604-1612

[10] Mao, X.: 'Stabilisation of continuous-time hybrid stochastic differential equations by discrete-time feedback control', Automatica, 2013, 49, (12), pp. 3677-3681

[11] Mao, X., Lam, J., Huang, L.: 'Stabilisation of hybrid stochastic differential equations by delay feedback control', Syst. Control Lett., 2008, 57, (11), pp. 927-935

[12] Mao, X., Matasov, A. and Piunovskiy, A.B.: 'Stochastic differential delay equations with Markovian switching', Bernoulli, 2000, 6, (1), pp. 73-90

[13] Mao, X., Yin, G., Yuan, C.: 'Stabilisation and destabilisation of hybrid systems of stochastic differential equations', Automatica, 2007, 43, (2), pp. 264-273

[14] Mao, X., Yuan, C.: 'Stochastic differential equations with Markovian switching' (Imperial College Press, 2006)

[15] Mariton, M.: 'Jump linear systems in automatic control' (Marcel Dekker, 1990)

[16] Mohammed, S.-E.A.: 'Stochastic functional differential equations' (Longman Scientific and Technical, 1986)

[17] Shi, P., Mahmoud, M.S., Yi, J., Ismail, A.: 'Worst case control of uncertain jumping systems with multi-state and input delay information', Information Sciences, 2006, 176, pp. 186-200

[18] Willsky, A.S., Levy,B.C.: 'Stochastic stability research for complex power systems' DOE Contract, LIDS, MIT, 1979, Report ET-76-C-01-2295.

[19] Wu, L., Shi, P., Gao, H.: 'State estimation and sliding mode control of Markovian jump singular systems', IEEE Trans. Autom. Control, 2010, 55, (5), pp. 1213-1219

[20] Wu, L., Su, X., Shi, P.: 'Sliding mode control with bounded $L_{2}$ gain performance of Markovian jump singular time-delay systems', Automatica, 2012, 48, (8), pp. 1929-1933

[21] Yin, G., Liu, R., Zhang, Q.: 'Recursive algorithms for stock liquidation: a stochastic optimisation approach', SIAM J. Optim., 2002, 13, (1), pp. 240-263

[22] Zhang, Q.: 'Stock trading: an optimal selling rule', SIAM J. Control Optim., 2001, 40, (1), pp. $64-87$ 